Pacific Journal of Mathematics

UNKNOTTING SPHERES VIA STALE 


\title{
UNKNOTTING SPHERES VIA SMALE
}

\author{
Rodolfo De SAPIO
}

It is shown here that a topological $n$-sphere which is embedded in Euclidean $m$-space $R^{m}$ with a transverse field of $(m-n)$-planes (in the sense of Whitehead) bounds a topological $(n+1)$-disc in $R^{m}$, provided $m>n+2>4$ and $n \neq 4$. On the other hand, Haefliger has constructed $C^{\infty}$ differentiable embeddings of the standard $(4 k-1)$-sphere $S^{4 k-1}$ in $6 k^{-}$-space $R^{6 k}$ which are differentiably knotted (i.e. they do not bound differentiably embedded $4 k$-discs in $R^{6 k}$ ). However, by using a sharpened form of the $h$-cobordism theorem of Smale it is possible to topologically unknot these spheres. This is achieved by showing that a differentiably knotted $n$-sphere in $m$-space $R^{m}$ is so knotted because of a single bad point (provided $m>n+2>4$ ). The topological case is then proved by first approximating the topologically embedded $n$-sphere by a differentiably embedded homotopy $n$-sphere, and thus reducing it to the differentiable case.

Differentiable or smooth will mean of class $C^{\infty}$. An $n$-disc is a contractible, compact, smooth $n$-manifold with simply connected boundary. A pair of disc $\left(B^{m}, B^{n}\right)$ is a pair of discs such that $\partial B^{n}=B^{n} \cap \partial B^{m}$, where $\partial M$ denotes the boundary of a manifold $M$, and where $B^{n}$ meets $\partial B^{m}$ transversally. A theorem of Smale [4] asserts that an $n$-disc for $n \geqq 6$ is diffeomorphic to the standard $n$-disc $D^{n}$ in $R^{n}$. Now let $\left(D^{m}, D^{n}\right)$ be the standard pair of discs.

Proposition 1. A pair of dises $\left(B^{m}, B^{n}\right)$ is diffeomorphic to the standard pair $\left(D^{m}, D^{n}\right)$, provided $m>n+2>7$.

Proof. This is an easy consequence of Smale [4; Corollary 3.2]. Let $\varphi:\left(D^{m}, D^{n}\right) \rightarrow\left(\operatorname{Int} B^{m}\right.$, Int $\left.B^{n}\right)$ be a smooth embedding and consider the exact homology sequence of the pair $\left(B^{n}-\operatorname{Int} \varphi\left(D^{n}\right), \varphi\left(\partial D^{n}\right)\right)$. By excision $H_{i}\left(B^{n}-\operatorname{Int} \varphi\left(D^{n}\right), \varphi\left(\partial D^{n}\right)\right) \approx H_{i}\left(B^{n}, \varphi\left(D^{n}\right)\right)=0$ and hence the the inclusion $\varphi\left(\partial D^{n}\right) \rightarrow B^{n}-\operatorname{Int} \varphi\left(D^{n}\right)$ is a homotopy equivalence. To show that the inclusion $\partial B^{n} \rightarrow B^{n}-\operatorname{Int} \varphi\left(D^{n}\right)$ is also a homotopy equivalence consider the homology sequence of the pair $\left(B^{n}-\operatorname{Int} \varphi\left(D^{n}\right), \partial B^{n}\right)$. By Poincaré duality

$$
H_{i}\left(B^{n}-\operatorname{Int} \varphi\left(D^{n}\right), \partial B^{n}\right) \approx H^{n-i}\left(B^{n}-\operatorname{Int} \varphi\left(D^{n}\right), \varphi\left(\partial D^{n}\right)\right)
$$

and by excision

$$
H^{n-i}\left(B^{n}-\operatorname{Int} \varphi\left(D^{n}\right), \varphi\left(\partial D^{n}\right)\right) \approx H^{n-i}\left(B^{n}, \varphi\left(D^{n}\right)\right) .
$$

Since $H^{n-i}\left(B^{n}, \varphi\left(D^{n}\right)\right)=0$, it follows that the inclusion $\partial B^{n} \rightarrow B^{n}-\operatorname{Int} \varphi\left(D^{n}\right)$ 
induces isomorphisms of homology and hence is a homotopy equivalence. Therefore, since $n>5$, [4; Corollary 3.2] implies that $B^{n}-\operatorname{Int} \varphi\left(D^{n}\right)$ is diffeomorphic to $S^{n-1} \times I$.

Similarly the inclusions $\varphi\left(\partial D^{m}\right) \rightarrow B^{m}-\operatorname{Int} \varphi\left(D^{m}\right)$ and $\partial B^{m} \rightarrow B^{m}-$ Int $\varphi\left(D^{m}\right)$ are homotopy equivalences and hence by [4; Corollary 3.2] the diffeomorphism $S^{n-1} \times I \approx B^{n}-\operatorname{Int} \varphi\left(D^{n}\right)$ may be extended to a diffeomorphism $S^{m-1} \times I \approx B^{m}-\operatorname{Int} \varphi\left(D^{m}\right)$, where, of course, $S^{n-1} \times I$ is embedded in $S^{m-1} \times I$ in the natural way. By using this product structure on $\left(B^{m}-\operatorname{Int} \varphi\left(D^{m}\right), B^{n}-\operatorname{Int} \varphi\left(D^{n}\right)\right)$ it is possible to define a diffeomorphism $\left(B^{m}, B^{n}\right) \approx\left(D^{m}, D^{n}\right)$, proving the proposition.

The following theorem is a slight generalization of the topological unknotting of a differentiably knotted $S^{n}$ in $S^{m}$ for $m>n+2>6$. Notice that Haefliger [1] has shown that $S^{n}$ differentiably knots in $S^{m}$ only if $3 n+3 \geqq 2 m \geqq 2 n+4$. Recall that a homotopy $n$-sphere is a closed, oriented, smooth $n$-manifold with the homotopy type of $S^{n}$.

THEOREM A. Any pair $\left(V^{m}, K^{n}\right)$ of homotopy spheres, with $m>n+2>6$, is diffeomorphic to a pair obtained from two copies of $\left(D^{m}, D^{n}\right)$ by identifying boundaries together through some diffeomorphism $\left(S^{m-1}, S^{n-1}\right) \rightarrow\left(S^{m-1}, S^{n-1}\right)$.

REMARK. If it is assumed that $K^{n}$ can be obtained by identifying two standard $n$-dises along their boundaries via a diffeomorphism $S^{n-1} \rightarrow S^{n-1}$, then the theorem is true for $n>3$.

Proof. The proof is simple; for $n \geqq 6$ even simpler. If $n \geqq 6$, choose an embedding $\varphi:\left(D^{m}, D^{n}\right) \rightarrow\left(V^{m}, K^{n}\right)$. By Proposition 1 the pair $\left(V^{m}-\operatorname{Int} \varphi\left(D^{m}\right), K^{n}-\operatorname{Int} \varphi\left(D^{n}\right)\right)$ is diffeomorphic to $\left(D^{m}, D^{n}\right)$. (It is easy to see that $\left(V^{m}-\operatorname{Int} \varphi\left(D^{m}\right), K^{n}-\operatorname{Int} \varphi\left(D^{n}\right)\right.$ is a pair of discs; for example, if $B^{n}=K^{n}-\operatorname{Int} \varphi\left(D^{n}\right)$, then by Poincaré duality $H_{i}\left(B^{n}-\varphi\left(\partial D^{n}\right) \approx H^{n-i}\left(B^{n}, \varphi\left(\partial D^{n}\right)\right)\right.$ and by excision $H^{n-i}\left(B^{n}, \varphi\left(\partial D^{n}\right)\right) \approx$ $H^{n-i}\left(K^{n}, \varphi\left(D^{n}\right)\right)$. Since $H^{n-i}\left(K^{n}, \varphi\left(D^{n}\right)\right) \approx H^{n-i}\left(K^{n}\right)$ for $i \neq n$, it follows that $B^{n}-\varphi\left(\partial D^{n}\right)$ is contractible and hence so is $B^{n}$.)

For $n=5$ choose disjoint smooth embeddings $\varphi_{i}: D^{5} \rightarrow K^{5}(i=1,2)$ so that $K^{5}-\operatorname{Int}\left[\varphi_{1}\left(D^{5}\right) \cup \varphi_{2}\left(D^{5}\right)\right]$ is diffeomorphic to $S^{4} \times I$ (this is possible because any homotopy 5 -sphere is, according to Milnor, $h$-cobordant to $S^{5}$ and hence, by Smale, is diffeomorphic to $\left.S^{5}\right)$. The embeddings $\varphi_{i}$ may be extended to smooth embeddings $\varphi_{i}:\left(D^{m}, D^{5}\right) \rightarrow\left(V^{m}, K^{5}\right)$ $(i=1,2)$. Now by the previous paragraph $V^{m}-\operatorname{Int} \varphi_{i}\left(D^{m}\right)$ is a disc and hence by the proof of Proposition 1 the $\varphi_{i}\left(\partial D^{m}\right)(i=1,2)$ are deformation retracts of $V^{m}-\operatorname{Int}\left[\varphi_{1}\left(D^{m}\right) \cup \varphi_{2}\left(D^{m}\right)\right]$. Therefore, by Smale [4; Corollary 3.2] the diffeomorphism $S^{4} \times I \approx K^{5}-\operatorname{Int}\left[\varphi_{1}\left(D^{5}\right) \cup \varphi_{2}\left(D^{5}\right)\right]$ may be extended to a diffeomorphism $S^{m-1} \times I \approx V^{m}-\operatorname{Int}\left[\varphi_{1}\left(D^{m}\right) \cup \varphi_{2}\left(D^{m}\right)\right.$, and the theorem then follows easily. 
Let $K^{n}$ be a homotopy $n$-sphere smoothly embedded in $S^{m}, m>n+2>6$, and let $\left(S^{m}, S^{n}\right)$ be the standard pair of spheres, $S^{n}$ embedded in $S^{m}$ by the natural inclusion of $R^{n+1}$ in $R^{m+1}$. A homeomorphism $f:\left(S^{m}, K^{n}\right) \rightarrow$ $\left(S^{m}, S^{n}\right)$ of pairs, differentiable except possibly at a single point of $K^{n}$, is obtained as follows: map one copy of the $\left(D^{m}, D^{n}\right)$ of Theorem A differentiably onto one pair of hemispheres of $\left(S^{m}, S^{n}\right)$ and then extend the map radially to the other copy of $\left(D^{m}, D^{n}\right)$ via the diffeomorphism $\left(S^{m-1}, S^{n-1}\right) \rightarrow\left(S^{m-1}, S^{n-1}\right)$ of Theorem A (i.e., the cone map) giving the diffeomorphism up to a point. Thus $f$ unknots $K^{n}$ in $S^{m}$.

COROLlaRY (Hirsch). Let $N$ be a closed tubular neighborhood of a homotopy $n$-sphere $K^{n}$ smoothly embedded in $S^{n+k}$. Then for $n \geqq 5$ and $k \geqq 3$ there is a diffeomorphism $N \approx S^{n} \times D^{k}$.

The closed tubular neighborhood $N$ is a neighborhood of $K^{n}$ in $S^{n+k}$ which is diffeomorphic to a neighborhood of the zero cross-section in the normal bundle of $K^{n}$ in $S^{n+k}$, the latter neighborhood being the set of all vectors less than or equal to some fixed $\varepsilon>0$. The following proof replaces the combinatorial arguments of Hirsch [3] by application of the above theorem.

Proof. Take a closed tubular neighborhood of $S^{n}$ in $S^{n+k}$; it is diffeomorphic to $S^{n} \times D^{k}$. It may be assumed that the closed normal tube $N$ is embedded in $S^{n} \times \operatorname{Int} D^{k}$ by the unknotting homeomorphism $f:\left(S^{m}, K^{n}\right) \rightarrow\left(S^{m}, S^{n}\right)$ constructed above. Moreover, $K$ may be deformed into $K^{\prime}$ by a differentiable isotopy deforming $N$ into a closed normal tube $N^{\prime}$ of $K^{\prime}$, where $N^{\prime} \subset$ Interior $N$ and $N^{\prime}$ does not contain the "bad point" of $f$. Then $N$ is diffeomorphic to $N^{\prime}$ and $N$ is smoothly embedded in $S^{n} \times \operatorname{Int} D^{k}$ by $f$. Now from an argument similar to that in Proposition 1 it follows that $\left(S^{n} \times D^{k}\right)-\operatorname{Int} f\left(N^{\prime}\right)$ is diffeomorphic to $S^{n} \times S^{k-1} \times I$. Consequently the boundary of $f\left(N^{\prime}\right)$ may be deformed isotopically onto $S^{n} \times S^{k-1}$. Since this isotopy may be extended to a differentiable isotopy deforming $f\left(N^{\prime}\right)$ onto $S^{n} \times D^{k}$, the corollary is proved.

REMARK. Theorem A implies that a smoothly embedded homotopy $n$-sphere $K^{n}$ in $S^{m}$, where $m>n+2>6$ is topologically unknotted. It can be shown that the pairs $\left(S^{m}, K^{n}\right)$ and $\left(S^{m}, S^{n}\right)$ may be smoothly triangulated so that the unknotting homeomorphism $f:\left(S^{m}, K^{n}\right) \rightarrow\left(S^{m}, S^{n}\right)$ is a combinatorial equivalence. More generally, however, Zeeman [7] has shown that a combinatorially embedded $S^{n}$ in $S^{m}$ is combinatorially unknotted if $m>n+2$. Stallings [5] proves that a locally flat $S^{n}$ in $S^{m}$ is unknotted if $n+3 \leqq m \geqq 5$.

Let $G_{m-n, n}$ be the Grassman manifold of $(m-n)$-planes in $R^{n}$. If $K^{n}$ is a topological $n$-manifold in $R^{m}, m>n>0$, then a field of 
$(m-n)$-planes transverse to $K^{n}$ (or a transverse field) is a continuous $\varphi: K^{n} \rightarrow G_{m-n, n}$ such that $\varphi(x)$ is transverse (in the sense of Whitehead [6]) to $K^{n}$ at $x$ for every $x \in K^{n}$. A topological $n$-manifold $K^{n}$ in $S^{m}$ is said to have a transverse field if $K^{n}$ has a transverse field in $S^{m}-\{\infty\}$ as defined above, where $\infty \in S^{m}-K$.

THeOREM B. A topological $n$-sphere $K^{n}$ embedded in $S^{m}$ with a transverse field unknots, provided $m>n+2>4$ and $n \neq 4$.

Of course $B$ follows from Stallings' result since such a $K^{n}$ is locally flat in $S^{m}$. In order to prove $B$ it is necessary to state some facts about transverse fields. So, suppose $K^{n}$ is a topological $n$-manifold in $R^{m}$ with a transverse field $\varphi: K \rightarrow G_{m-n, n}$. The space

$$
E(\varphi)=\{(x, y) \mid x \in K, y \in \varphi(x)\}
$$

may be considered as the total space of the $(m-n)$-plane bundle over $K$ induced by $\varphi$; the fibre over $x \in K$ is the $(m-n)$-plane $\varphi(x)$. Now by Whitehead [6; page 157, second sentence], given a continuous map $\varepsilon: K \rightarrow R_{+}\left(R_{+}\right.$the positive reals), there is a Lipschitz map $\phi^{\prime}: K \rightarrow G_{m-n, n}$ which is an $\varepsilon$-approximation to $\varphi$, and by [6; Theorem 1.3] $\varepsilon$ may be chosen so that $\varphi^{\prime}$ is a transverse field (which is transversally homotopic to $\varphi$ ). Hence we may assume without loss of generality that the given transverse field $\varphi$ is Lipschitz.

Define a map

$$
\theta: E(\varphi) \rightarrow R^{m}
$$

by $\theta(x, y)=x+y$. By [6; Theorem 1.5] there exists a map $\rho: K \rightarrow R_{+}$ ( $R_{+}$the positive reals) such that if

$$
T_{\rho}^{\prime}=\{(x, y)|(x, y) \in E(\varphi),| y \mid<\rho(x)\},
$$

an open subset of $E(\phi)$, then $\theta \mid T_{\rho}^{\prime}$ is a regular Lipschitz homeomorphism of $T_{\rho}^{\prime}$ onto $\theta T_{\rho}^{\prime}$. Now define the $\varphi$-projection $\pi$ of $\theta T_{\rho}^{\prime}$ onto $K$ by

$$
\pi \theta(x, y)=\pi(x+y)=x .
$$

Then $\varphi$ is said to be of class $C^{r}(1 \leqq r \leqq \infty)$ if $\varphi \pi$ is of class $C^{r}$ in a neighborhood $N \subset \theta T_{\rho}^{\prime}$ of $K$. In this case by [6; Theorem 3] there exists a smooth $C^{r}$ submanifold $M^{n}$ of $N$ such that $\pi \mid M: M \rightarrow K$ is a homeomorphism and the map $M \rightarrow G_{m-n, n}$ sending $x$ into $\varphi \pi(x)$ is a transverse field on $M$.

Theorem $\mathrm{B}$ is a direct consequence of Theorem $\mathrm{A}$ (for $n=3$ see Remark after Theorem A) and the following.

Proposition 2. A pair $\left(S^{m}, K^{n}\right)$, where $K^{n}$ is a closed topological manifold in $S^{m}$ with a transverse field $\varphi: K \rightarrow G_{m-n, n}$, is homeomorphic to a pair $\left(S^{m}, M^{n}\right)$, where $M^{n}$ is a smooth $C^{\infty}$ submanifold of $S^{m}$. 
REMARK. The homeomorphism of the pairs $\left(S^{m}, K^{n}\right)$ and $\left(S^{m}, M^{n}\right)$ which is defined in the following proof is isotopic (homotopic through homeomorphisms) to the identity map of $S^{m}$.

Proof. Let $\rho: K \rightarrow R_{+}$be as above; by [6; Theorem 1.10] $\varphi$ may be assumed to be a $C^{\infty}$ transverse field. Now choose $\rho_{0}>0$ such that $0<\rho_{0}<\operatorname{Inf}\{\rho(x) \mid x \in K\}$ and let

$$
T_{0}^{\prime}=\left\{(x, y) \in E(\varphi)|| y \mid<\rho_{0}\right\}, T_{0}=\theta T_{0}^{\prime} .
$$

Clearly $T_{0}^{\prime} \subset T_{\rho}^{\prime}$ and, moreover, the map $\psi: T_{0} \rightarrow E(\varphi)$ sending $x+y \rightarrow$ $\left(x,\left(1 /\left(\rho_{0}-|y|\right)\right) y\right)$ defines a homeomorphism of

$$
T_{0}=\left\{x+y|x \in K, y \in \varphi(x),| y \mid<\rho_{0}\right\} \quad \text { onto } E(\varphi) .
$$

By remarks above there exists a smooth $C^{\infty}$ submanifold $M^{n}$ of $R^{m}$ in $T_{0}$ such that $\pi \mid M: M \rightarrow K$ is a homeomorphism. The homeomorphism $\pi \mid M$ will be extended to a surjective homeomorphism $f: S^{m} \rightarrow S^{m}$. The first step is to extend $\pi \mid M$ to a homeomorphism $\bar{\pi}: T_{0} \rightarrow T_{0}$ onto $T_{0}$ in the following way: the image of $M$ under $\psi: T_{0} \rightarrow E(\phi)$ may be described as the set $\left\{(x, \alpha(x)) \mid x \in K, \alpha(x) \in \varphi(x), \alpha: K \rightarrow R^{m}\right\}$ and so the map $\beta: E(\phi) \rightarrow E(\varphi)$ defined by $\beta(x, y)=(x, y-\alpha(x))$ is clearly a homeomorphism of $E(\varphi)$ onto itself. Setting $\bar{\pi}=\psi^{-1} \beta \psi$ gives the desired extension of $\pi \mid M$.

It is a tedious but straightforward verification that for $(x+y) \in T_{0}$, $|\bar{\pi}(x+y)-(x+y)| \rightarrow 0$ uniformly for all $x$ as $|y| \rightarrow \rho_{0}$ and hence by defining $f: S^{m} \rightarrow S^{m}$ to be, for each $s$ in $S^{m}$,

$$
f(s)= \begin{cases}\bar{\pi}(s) & \left(\text { if } s \in T_{0}\right), \\ s & \left(\text { if } s \notin T_{0}\right),\end{cases}
$$

it follows that $f$ is a homeomorphism of $S^{m}$ onto $S^{m}$ sending $M$ onto $K$.

\section{REFERENCES}

1. A. Haefiiger, Plongements différentiable de variétés dans variétés, Comment. Math. Helv. 36 (1961), 47-82.

2. - Knotted (4k-1)-spheres in 6k-space, Ann. of Math. 75 (1962), 462-466.

3. M. W. Hirsch, Smooth regular neighborhoods, Ann. of Math. 76 (1962), 524-530.

4. S. Smale, On the structure of manifolds, Arner. J. Math. 84 (1962), 387-399.

5. J. Stallings, On topologically unknotted spheres, Ann. of Math. 77 (1963), 490-503.

6. J. H. C. Whitehead, Manifolds with transverse fields in Euclidean space, Ann. of Math. 73 (1961), 154-212.

7. E. C. Zeeman, Unknotting combinatorial balls, Ann. of Math. 78 (1963), 501-526.

Received March 3, 1965. This research was partially supported by National Science Foundation Grant No. 4069. 



\section{PACIFIC JOURNAL OF MATHEMATICS}

H. SAMELSON

Stanford University Stanford, California

R. M. BLUMENTHAL

University of Washington

Seattle, Washington 98105

\section{EDITORS}

\author{
*J. DugundJI \\ University of Southern California \\ Los Angeles, California 90007 \\ RICHARD ARENS \\ University of California \\ Los Angeles, California 90024
}

\section{ASSOCIATE EDITORS}

E. F. BeCKENBACH

B. H. NEUMANN

F. WOLF

K. YosidA

\section{SUPPORTING INSTITUTIONS}

UNIVERSITY OF BRITISH COLUMBIA

CALIFORNIA INSTITUTE OF TECHNOLOGY

UNIVERSITY OF CALIFORNIA

MONTANA STATE UNIVERSITY

UNIVERSITY OF NEVADA

NEW MEXICO STATE UNIVERSITY

OREGON STATE UNIVERSITY

UNIVERSITY OF OREGON

OSAKA UNIVERSITY

UNIVERSITY OF SOUTHERN CALIFORNIA
STANFORD UNIVERSITY

UNIVERSITY OF TOKYO

UNIVERSITY OF UTAH

WASHINGTON STATE UNIVERSITY

UNIVERSITY OF WASHINGTON

AMERICAN MATHEMATICAL SOCIETY CHEVRON RESEARCH CORPORATION TRW SYSTEMS

NAVAL ORDNANCE TEST STATION 


\section{Pacific Journal of Mathematics}

Vol. 18, No. 1

March, 1966

Edward Joseph Barbeau, Semi-algebras that are lower semi-lattices ...... 1

Steven Fredrick Bauman, The Klein group as an automorphism group

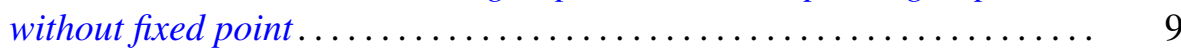

Homer Franklin Bechtell, Jr., Frattini subgroups and $\Phi$-central groups .... 15

Edward Kenneth Blum, A convergent gradient procedure in prehilbert

spaces ............................................

Edward Martin Bolger, The sum of two independent exponential-type random variables ...................................

David Wilson Bressler and A. P. Morse, Images of measurable sets .......

Dennison Robert Brown and J. G. LaTorre, A characterization of uniquely

divisible commutative semigroups........................

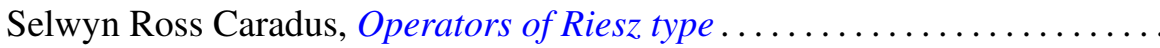

Jeffrey Davis and Isidore Isaac Hirschman, Jr., Toeplitz forms and ultraspherical polynomials ............................

Lorraine L. Foster, On the characteristic roots of the product of certain rational integral matrices of order two ......................

Alfred Gray and S. M. Shah, Asymptotic values of a holomorphic function

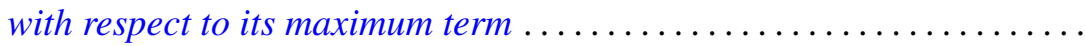

Sidney (Denny) L. Gulick, Commutativity and ideals in the biduals of

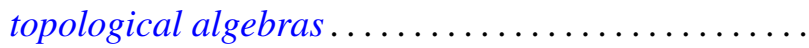

G. J. Kurowski, Further results in the theory of monodiffric functions

Lawrence S. Levy, Commutative rings whose homomorphic images are self-injective .

Calvin T. Long, On real numbers having normality of order $k$....

Bertram Mond, An inequality for operators in a Hilbert space. ...

John William Neuberger, The lack of self-adjointness in three-point boundary value problems ........................

C. A. Persinger, Subsets of $n$-books in $E^{3}$

Oscar S. Rothaus and John Griggs Thompson, A combinatorial problem in the symmetric group ............................... 175

Rodolfo DeSapio, Unknotting spheres via Smale .................. 179

James E. Shockley, On the functional equation

$$
F(m n) F((m, n))=F(m) F(n) f((m, n)) \ldots \ldots \ldots
$$

Kenneth Edward Whipple, Cauchy sequences in Moore spaces ... 